\title{
Watching a real moving object expands tactile duration: The role of task-irrelevant action context for subjective time
}

\author{
${\text { Lina } \mathrm{Jia}^{1} \cdot \text { Zhuanghua Shi }^{2,4} \cdot \text { Xuelian Zang }}^{2} \cdot$ Hermann J. Müller ${ }^{2,3}$
}

Published online: 15 August 2015

(C) The Psychonomic Society, Inc. 2015

\begin{abstract}
Although it is well established that action contexts can expand the perceived durations of action-related events, whether action contexts also impact the subjective duration of events unrelated to the action remains an open issue. Here we examined how the automatic implicit reactions induced by viewing task-irrelevant, real moving objects influence tactile duration judgments. Participants were asked to make temporal bisection judgments of a tactile event while seeing a potentially catchable swinging ball. Approaching movement induced a tactile-duration overestimation relative to lateral movement and to a static baseline, and receding movement produced an expansion similar in duration to that from approaching movement. Interestingly, the effect of approaching movement on the subjective tactile duration was greatly reduced when participants held lightweight objects in their hands, relative to a hands-free condition, whereas no difference was obtained in the tactile-duration estimates between static hands-free and static hands-occupied conditions. The results indicate that duration perception is determined by internal bodily states as well as by sensory evidence.
\end{abstract}

Zhuanghua Shi

strongway@psy.lmu.de

School of Humanities, Jiangnan University, Wuxi, China

2 Experimental Psychology, Ludwig-Maximilians-Universität München, Munich, Germany

3 School of Psychological Science, Birkbeck College, University of London, London, UK

4 Experimental Psychology, Department of Psychology, Ludwig-Maximilians-Universität München, 80802 Munich, Germany
Keywords Duration estimation · Bodily states · Embodiment - Crossmodal interaction - Temporal processing . Embodied perception

Time perception in the subsecond to second ranges is susceptible to a wide range of factors (for a review, see Shi, Church, \& Meck, 2013), such as the contrast and arousal properties of the stimuli (Droit-Volet \& Gil, 2009; Grondin, Laflamme, \& Gontier, 2014; Matthews, 2011; Shi, Jia, \& Müller, 2012), repetition of the presentation (Pariyadath \& Eagleman, 2008), and voluntary action (Morrone, Ross, \& Burr, 2005; Yarrow, Haggard, Heal, Brown, \& Rothwell, 2001). Most of us are likely to have experienced "chronostasis" (Yarrow et al., 2001), an illusion of time in which a clock's second hand appears to stand still when one directs one's gaze from elsewhere onto it; that is, the first second that you see the clock tick appears to be longer than the subsequent seconds. Similar time distortions have been reported for various other action contexts. For example, a voluntary keypress can expand the perceived duration of a stimulus triggered by it (Park, SchlagRey, \& Schlag, 2003), and an event is perceived as longer when it relates to an action goal and occurs during the preparation of the action (Hagura, Kanai, Orgs, \& Haggard, 2012). Recent studies have shown that even the action meanings of static pictures can engender distortions of their perceived durations (Gable \& Poole, 2012; Nather, Bueno, Bigand, \& Droit-Volet, 2011; Orgs, Bestmann, Schuur, \& Haggard, 2011; Yamamoto \& Miura, 2012).

Several accounts have been developed to explain the effects of action on time perception. The original account suggested for the chronostasis illusion was sensorimotor interference (Yarrow et al., 2001) - that is, that the time distortion arises primarily from interference of the action with the processing of sensory inputs. In a typical chronostasis illusion, 
saccadic eye movements blur the onset of a visual target that appears while the saccade is being executed, causing the brain to backdate the onset of the target to the saccadic onset (Yarrow, Haggard, \& Rothwell, 2004). A second account suggests that arousal induced by the action could be the main factor for the time distortion, since actions are often coupled with changes of arousal (Hodinott-Hill, Thilo, Cowey, \& Walsh, 2002). The arousal account draws on well-developed "internal clock" models (Gibbon \& Church, 1990; Gibbon, Church, \& Meck, 1984; Shi, Ganzenmüller, \& Müller, 2013; Zakay \& Block, 1997), which generally assume three essential stages of time processing: a clock stage, at which a pacemaker generates pulses that are transmitted to an accumulator through an on/off switch; a memory stage, during which the accumulated pulses are transferred to working memory as a measure of the target interval; and a decision stage, at which the time representation from working memory is compared to a representation from reference memory. An action or action context may arouse the internal clock system, speeding up the pacemaker and shortening the latency of the switch, resulting in an expansion of the subjective duration (Hodinott-Hill et al., 2002; Nather et al., 2011). Note, however, that not all actions or action contexts are sufficiently arousing to cause a reliable distortion of subjective duration (Yarrow et al., 2004). Moreover, in contrast to speeding up the internal clock, actions such as saccadic eye movements have also been suggested to slow down the internal clock, giving rise to time compression (Morrone et al., 2005).

Recently, an alternative, embodied view has been emerging to account for the influences of action or action context on time perception - proposing that time is not a pure perceptual phenomenon, but can only be fully understood by taking the context of the perception-action loop into consideration (Craig, 2009; Droit-Volet, Fayolle, Lamotte, \& Gil, 2013; Effron, Niedenthal, Gil, \& Droit-Volet, 2006; Niedenthal, 2007; Wittmann, 2013; Wittmann \& van Wassenhove, 2009). The notion of a tight linkage between perception and action is central to embodied cognition (e.g., see Clark, 1999; Engel, Maye, Kurthen, \& Konig, 2013), which fundamentally assumes that perceptual and cognitive processes are subject to the constraints of external stimuli on actions. A classic study of emotion and time perception provides a nice demonstration of how embodiment influences duration judgments (DroitVolet \& Meck, 2007; Effron et al., 2006). Individuals watched an affective face-for instance, an angry or a neutral faceand were asked to judge the duration for which the face was presented. Automatic adoption ("imitation") of the affective facial expressions distorted the subjective duration of the facial stimuli, giving rise to a duration expansion for angry, as compared to neutral, faces. Interestingly, the subjective expansion for angry faces diminished when imitation of the facial expressions was inhibited by participants holding a pen between their lips (Effron et al., 2006). Another recent study
(Gable \& Poole, 2012) also revealed that a stimulus that evokes a positive, approach motivation (e.g., a delicious dessert) shortens its perceived time; the authors attributed this to the possibility that the shortening of subjective durations in an appetitive state would prolong the pursuit of appetitive objects or goals.

It should be pointed out, though, that the arousal and embodiment accounts are not mutually exclusive. Both acknowledge an important role of the affective system in time perception, with the embodied view highlighting more general influences of bodily states and sensorimotor activation. A recent study has suggested that action meanings may have a greater impact on crossmodal duration judgments than basic affective stimulus evaluations in terms of valence and arousal properties (Shi et al., 2012). In that study, participants were asked to judge a tactile duration after viewing an affective picture. They tended to judge the tactile duration to be longer after exposure to an affective picture that engendered threat meanings, as compared to disgust meanings, even though both types of pictures had similar affective properties (arousal and valence). Shi et al. (2012) took this finding to suggest that viewing a picture with a threat meaning automatically activates the defensive system, which then causes a subjective expansion of tactile stimuli that are sensed by the activated sensorimotor system.

From a theoretical point of view, it is worth noting that the studies mentioned above only investigated situations in which the actions or action contexts were highly relevant to the task of estimating durations. By contrast, little is known as yet about how task-irrelevant or timing-irrelevant action contexts (i.e., contexts entirely unrelated to the to-be-judged stimuli/ events) influence duration estimation. This question is arguably important because, although timing-relevant and irrelevant action contexts induce similar motor activations, they may have very different impacts on subjective timing. In particular, timing-irrelevant action contexts (in contrast to relevant contexts) may divert attention away from temporal processing. According to "attentional-gate" theory (Zakay \& Block, 1997), allocating attention to a nontiming task would temporally halt the timing process at the clock stage, resulting in an underestimation of the duration of the to-be-judged stimulus. The arousal account (Hodinott-Hill et al., 2002), on the other hand, predicts either an unchanged or an overestimated target duration, dependent on how arousing the task-irrelevant action context is. In contrast, the notion of embodied timing (Craig, 2009; Droit-Volet et al., 2013; Effron et al., 2006), also advocated by us (Shi et al., 2012), predicts that task-irrelevant action contexts may alter bodily states and thus facilitate sensorimotor processing (Hagura et al., 2012), which in turn would lead to an overestimation of the target duration.

The present study was designed to examine whether and how task-irrelevant action contexts influence subjective duration. Many behaviors, such as the automatic evasion of fast- 
approaching objects, arise as a result of stimulus-response (S-R) associations acquired through experience in our daily activities. Such associations can automatically evoke motor activation, even when individuals have no intention of making the movement concerned (Fotowat \& Gabbiani, 2011; Tucker \& Ellis, 2004). Accordingly, we used a real moving object as a task-irrelevant action context to "simulate" automatic S-R linkages in the real world. In particular, in the present study, participants were asked to judge the duration of a tactile vibration delivered to a fingertip while they were seeing a real moving object, a small metal ball. If the action context induced by automatic S-R associations activated by the ball movement increased the separable "moment" (count) of bodily states, the duration of the concurrent tactile event might be overestimated, indicative of a subjective expansion of time. Furthermore, the magnitude of the duration expansion might critically depend on the potential for interaction with the moving object, such as whether the object would, or would not, be easy to catch. To address this question, we first examined the influences of approaching and lateral ball movements on perceived tactile durations, given that an approaching ball might be expected to induce stronger $\mathrm{S}-\mathrm{R}$ associations than would a lateral movement or a static baseline condition (Exp. 1). However, visual looming in the approaching movement as a salient signal might also potentially contribute to the duration distortion. To control for this and to disentangle the effects of visual looming on subjective duration from those of an implicit interaction, we compared and contrasted the impacts of approaching and receding movements in Experiment 2. Task-irrelevant action contexts could also generate shifts of attention and changes in arousal states, which might also alter time judgments. One distinguishing prediction of the embodied view is that subjective duration expansion would be greatly reduced or even reversed when activation of an embodied reaction was prevented (Effron et al., 2006). On this basis, we hypothesized that the S-R linkage would be weakened (to some degree) when the observer was holding an object in his or her hand. If so, approaching ball movements would have less impact on vibrotactile duration judgments for the hands-occupied (i.e., holding objects) than for the hands-free condition. Alternatively, if attention and/or arousal states are the determining factors, subjective duration judgments should not differ between the two hand state conditions. Accordingly, in Experiments 3 and 4, we presented participants with approaching movements and static conditions while they held two lightweight objects in their hands (intended to inhibit implicit reactions to the ball). Experiment 3 involved a Movement (approaching vs. static) $\times$ Hand State (hands free vs. occupied) manipulation to exclude possible influences of affordances and attention on tactile duration judgments. In the subsequent Experiment 4, to rule out that the tactile duration judgments in Experiment 3 were modulated by arousal, we measured arousal levels by means of skin conductance responses and compared the results among the approaching-movement hands-occupied, approaching-movement hands-free, and static baseline conditions.

\section{General method}

\section{Participants}

A total of 41 participants took part in the Experiments 1, 2, 3, and 4 (11 in Exp. 3, and 10 in each of the other experiments; 26 female, 15 male; mean age 22 years). All participants had normal or corrected-to-normal vision and no somatosensory disorders. They gave written informed consent prior to the experiment and were paid for their service.

\section{Stimuli and apparatus}

All four experiments were conducted in a sound-isolated and dimly lit $\left(0.76 \mathrm{~cd} / \mathrm{m}^{2}\right)$ cabin. Vibrotactile $(200-\mathrm{Hz})$ stimuli were produced by a vibrator (AEC Tactaid VBW32) and delivered to the participant's right-hand index finger. To avoid vibrotactile stimulation being heard, participants wore a noisecancelling headphone during each block. A simple pendulum was constructed of a metal ball ( $2 \mathrm{~cm}$ in diameter) suspended on a string $(108 \mathrm{~cm})$ from the ceiling of the cabin. At its resting position, the ball was $4.5 \mathrm{~cm}$ above the table at which the participant was seated; a black fixation cross marked the table right below this position. For "static" trials (see the Design and Procedure section below), the ball was kept static at the resting position, whereas the ball swung back and forth for one cycle $(2 \mathrm{~s})$ for the "movement" trials, with swing amplitudes (i.e., the ball's maximum angular displacement from its equilibrium position) of $27^{\circ}, 20^{\circ}, 20^{\circ}$, and $20^{\circ}$, in Experiments 1, 2, 3, and 4, respectively (see Fig. 1a). Note that in Experiments 3 and 4, two lightweight objects (black cylinders, $2.5 \mathrm{~cm}$ in height and $4.2 \mathrm{~cm}$ in diameter, weighing $93 \mathrm{~g}$ ) were placed in participants' hands in order to inhibit interaction. In Experiment 4, skin conductance responses (SCRs) were recorded additionally, at a sampling rate of $500 \mathrm{~Hz}$ using a BioPac system (MP35); the two SCR sensors were attached to participants' left-hand index and middle fingers.

\section{Design and procedure}

Participants were asked to place their hands comfortably on the table in palm-up position, and the tactile vibrator was attached to the participant's right index finger using an elastic finger sleeve. The hand positions relative to the ball's movement trajectories are illustrated in Fig. 1b-d. A temporal 
bisection task, which required participants to classify the vibrotactile duration presented on a trial into the "short" or the "long" category, was used in all experiments. At the beginning of the experiment, participants were first trained to discriminate two vibrotactile standard durations, one short (200 ms) and one long (600 ms), until they could perfectly classify the two standards. Then, in the formal test session, a probe vibrotactile stimulus, with a duration randomly selected from 200, 300, 400, 500, and $600 \mathrm{~ms}$ on each test trial, was delivered to the participant, who was asked to indicate whether the presented vibrotactile duration was closer to the short or to the long standard, by pressing either the left or the right foot pedal.

During the test session, we manipulated the ball movements, which were totally irrelevant to the temporal bisection task, in three conditions: approaching, lateral, and "static" in Experiment 1 (Fig. 1b); and approaching, receding, and "static" in Experiment 2 (Fig. 1c). Next, in Experiment 3 (Fig. 1d), we examined tactile duration judgments in 2 (Movement: approaching vs. static) $\times 2$ (Hand State: hands free vs. hands occupied) combined conditions. The approaching-movement and static conditions with free hands were the same as in the previous experiments; in the handsoccupied conditions, two lightweight cylinders were placed in the palms of the participants' left and right hands. In Experiment 4 (Fig. 1d), we compared duration judgments among three conditions: (i) approaching-ball, hands-free; (ii) approaching-ball, hands-occupied; and (iii) static ball, with half of the trials hands-free and half hands-occupied.

At the beginning of trials with ball movements, the experimenter initiated a pendulum movement by releasing the ball (initially held) in his or her right hand and simultaneously released a mouse button (initially pressed) with the left hand, with the button release serving as a measure of the onset of the swing. ${ }^{1}$ A vibration with a maximum duration of $600 \mathrm{~ms}$, with its onset at a random point (within 200-400 ms) relative to the start of the swing (marked by the release of the mouse button), was completely delivered to the participant's index finger during the first half cycle of the pendulum swing. Stimulus presentation was ended once the pendulum had swung back (completing the full cycle) and was quietly grasped by the experimenter. During the trial, participants were instructed to fixate their eyes on the ball in front of them. On "static" trials, the ball stayed in the resting position, and the experimenter lifted the finger to release the mouse button, which was visible

\footnotetext{
${ }^{1}$ The temporal discrepancy between the experimenter's left- and righthand releases was small: Measuring the discrepancy, across 100 trials prior to the experiment, between releasing a response button of the parallel-port keypad with one hand and releasing a mouse button with the other hand simultaneously yielded a mean difference of $3 \mathrm{~ms}$, with a standard deviation of $12 \mathrm{~ms}$ and a maximum difference of $42 \mathrm{~ms}$ - which can be regarded as a randomly jittered onset of the swing relative to the $2 \mathrm{~s}$ of the whole cycle of the pendulum swing.
}

to the participants, so as to initiate the trial. The onset of the vibration was triggered randomly 200-400 ms after the button release. The experimental conditions were tested block-wise in a random order, in order to avoid the experimenter having frequently to change his or her position. At the beginning of each block, five reminder trials with the short and long vibrotactile stimuli (200 and $600 \mathrm{~ms}$, respectively) were presented to refresh the participant's memory of the two standards. Experiments 1, 2, and 4 consisted of 12 blocks, each with 25 trials, and Experiment 3 consisted of 16 blocks of 25 trials. Each experimental condition was repeated 20 times.

After the formal test session in Experiment 4, participants were asked to rate the valence and arousal of the movement using the 9-point affective-rating Self-Assessment Manikin (SAM; Bradley \& Lang, 1994), in order to assess whether the arousal levels were comparable between the hands-free and hands-occupied approaching-movement conditions. Prior to the ratings, participants were provided with detailed instructions as to the meanings of the nine points on the valence and arousal scales.

\section{Data analysis}

For the temporal bisection task, we first calculated the proportions of "long" responses for the five probe durations (200, $300,400,500$, and $600 \mathrm{~ms}$ ), separately for each participant and condition, and fitted psychometric curves to these proportions using logistic regression. From the fitted curves, the points of subjective equality (PSEs) of the temporal bisection were then estimated as the respective durations corresponding to the $50 \%$ threshold, to provide an indicator of the subjective bisection point between 200 and $600 \mathrm{~ms}$. In a similar way, the just-noticeable differences (JNDs) were calculated, by taking half of the difference in duration between the $25 \%$ and $75 \%$ thresholds, in order to provide an estimate of discrimination sensitivity. In Experiment 4, SCRs were measured in microohms to monitor arousal changes, and log transformation (log $[\mathrm{SCR}+1])$ was used to normalize the distributions. Changes in SCRs were calculated after baseline correction ( $2 \mathrm{~s}$ before stimulus onset) for the time window from 1 to $4 \mathrm{~s}$ post-stimulus-onset.

Repeated measures analyses of variance (ANOVAs) were conducted separately on the PSEs and JNDs, with Type of Ball Movement as factor in all experiments. Mauchly's tests of sphericity, applied for deciding on the "correct" $F$ and $p$ values, revealed no significant violations of sphericity for the PSEs and JNDs (all $p s>.05$ ) in Experiments 1, 2, and 4. Multiple-comparison tests were corrected by the HolmBonferroni method to ascertain significant differences among the critical experimental conditions. To provide further informative results, scaled Jeffrey-Zellner-Siow (JZS) Bayes factors are reported. Repeated measures ANOVAs were also 
applied to the SAM ratings and to the physiological (SCR) data from Experiment 4.

\section{Results and discussion}

\section{Experiment 1}

In this experiment, we examined the influences of approaching and lateral irrelevant movements on vibrotactile temporal bisection performance. Figure 2 shows the psychometric curves of the tactile-duration bisection task for the three ball-movement conditions. The mean PSEs ( $\pm 95 \%$ confidence intervals [CIs]) were $360 \pm 14,376 \pm 13$, and $400 \pm$ $21 \mathrm{~ms}$, respectively, for the approaching-movement, lateralmovement, and static baseline conditions, revealing a significant influence of the ball movements on the tactile-duration judgments, $F(2,18)=10.04, p<.01, \eta_{\mathrm{p}}^{2}=.53$. Follow-up Holm-Bonferroni corrections confirmed that the mean PSEs were significantly lower for the approaching-movement than for the lateral-movement and baseline conditions (mean differences: $16 \mathrm{~ms}$, corrected $p=.02$; and $40 \mathrm{~ms}$, corrected $p=$ $.00)$, and were marginal lower for the lateral-movement than for the baseline condition (mean difference: $24 \mathrm{~ms}$, corrected $p=.05$ ). Lower-than-baseline PSEs mean more "long" responses, indicative of a subjective duration expansion. Similarly, scaled JZS Bayes factor (B.F.) analyses favored the alternative hypothesis for the three pairs of conditions. Specifically, the differences were substantial between the approaching- and lateral-movement conditions (JZS B.F. = 0.17), strong between the approaching-movement and baseline conditions (JZS B.F. $=0.08$ ), and anecdotal between the lateral-movement and baseline conditions (JZS B.F. $=0.53$ ). The results of Experiment 1 demonstrate that the vibrotactile duration was significantly overestimated in the approachingmovement condition relative to the static-baseline condition, and thus favor a difference between the approaching- and lateral-movement conditions.

In contrast to the PSE, discrimination sensitivity measured by the JNDs (or the slopes of the psychometric functions) did not differ significantly among the three conditions, $F(2,18)=$ $1.59, p=.23, \eta_{\mathrm{p}}{ }^{2}=.15$. This suggests that the ball movement did not affect the sensitivity of temporal bisection performance.

\section{Experiment 2}

Experiment 1 demonstrated marked duration overestimation in the approaching-movement condition, which could be attributable to either a visual-looming or an arousal effect (Droit-Volet \& Meck, 2007; van Wassenhove, Wittmann, Craig, \& Paulus, 2011; Wittmann, van Wassenhove, Craig, \& Paulus, 2010). Previous studies have shown that looming signals, as compared to receding and static signals, are particularly salient events that, as such, might have given rise to the relatively pronounced duration overestimation (van Wassenhove et al., 2011; Wittmann, van Wassenhove, et al., 2010). Thus, quite possibly, different types of visual movement, such as approaching and receding stimuli, might have differential effects on tactile duration judgments. Experiment 2 was designed to examine this prediction by comparing the vibrotactile temporal bisection judgments among the approaching-movement, receding-movement, and static baseline conditions.

Again, the ANOVA revealed a significant influence of the ball movement on the tactile duration judgments, $F(2,18)=$ $7.29, p<.01, \eta_{\mathrm{p}}{ }^{2}=.45$. The mean PSEs $( \pm 95 \% \mathrm{CIs})$ were 380 $\pm 19,381 \pm 25$, and $407 \pm 22 \mathrm{~ms}$ for the approaching-movement, receding-movement, and static baseline conditions, respectively (see Fig. 3). Post-hoc Holm-Bonferroni correction revealed that the PSEs were significantly lower in both the approaching- and receding-movement conditions than for the static baseline (mean differences: $27 \mathrm{~ms}$, corrected $p<$ $.001 ; 26 \mathrm{~ms}$, corrected $p=.02$ ), without any difference between the two movement conditions (corrected $p=.95$ ). Consistent with the Holm-Bonferroni results, scaled JZS B.F.s favored strong differences between the approachingmovement and static conditions (JZS B.F. $=0.08$ ) and between the receding-movement and static conditions (JZS B.F. $=0.10$ ), but favored the null hypothesis between the approaching- and receding-movement conditions (JZS B.F. $=3.23$ ). This pattern indicates that the overestimation of tactile duration is not attributable to the particular type of stimulus movement; rather, it is likely due to an implicit reaction induced by both types of moving object.

As in Experiment 1, the JNDs did not differ among the three conditions, $F(2,18)=0.92, p=.42, \eta_{\mathrm{p}}{ }^{2}=.09$, suggesting that the pendulum movement did not change the temporal discrimination sensitivity.

\section{Experiment 3}

In Experiment 2, the overestimation was observed even in the receding condition, which effectively ruled out an influence of visual looming in the action context on tactile duration judgments. To further test the embodied view of time perception, we examined whether the duration expansion would be greatly reduced when participants' hands were occupied in the approaching condition. In addition, to rule out a potential confounding of attention and affordance, we implemented a full factorial design: Hand State (hands-occupied vs. hands-free) $\times$ Ball State (approaching vs. static).

The mean PSEs ( $\pm 95 \%$ CIs) were $397 \pm 27,418 \pm 24,415$ \pm 17 , and $410 \pm 17 \mathrm{~ms}$ for the approaching-movement handsfree, approaching-movement hands-occupied, static handsfree, and static hands-occupied conditions, respectively. A two-way repeated measures ANOVA with the factors Ball 
State (approaching vs. static) and Hand State (hands free vs. occupied) failed to reveal any significant main effects: $F(1$, $10)=0.18, p=.68, \eta_{\mathrm{p}}{ }^{2}=.07$, for ball state, and $F(1,10)=$ $2.66, p=.13, \eta_{\mathrm{p}}{ }^{2}=.32$, for hand state. However, interestingly, their interaction was significant, $F(1,10)=5.26, p<.05, \eta_{\mathrm{p}}{ }^{2}=$ .54 (see Fig. 4). As can be seen from Fig. 4, the interaction was mainly caused by marked changes of the PSEs in two approaching conditions. The Holm-Bonferroni comparison tests revealed a significant difference between the approachingmovement hands-free and approaching-movement hands-occupied conditions (mean difference: $21 \mathrm{~ms}$, corrected $p=.02$ ), but not between the static hands-free and static handsoccupied conditions (mean difference: $5 \mathrm{~ms}$, corrected $p=$ .58). Scaled JZS B.F.s confirmed the strong difference between the two approaching-movement conditions (JZS B.F. $=0.10$ ), but favored no difference between the two static conditions (JZS B.F. = 2.93). This pattern suggests that the hand states may play a (perhaps important) role for vibrotactile duration judgments when a potentially graspable moving object is presented.

The ANOVA of the JNDs once again yielded no significant effects at all [ball state, $F(1,10)=0.03, p=.86, \eta_{\mathrm{p}}{ }^{2}=.05$; hand state, $F(1,10)=0.16, p=.70, \eta_{\mathrm{p}}{ }^{2}=.07$; interaction, $F(1,10)=$ $\left.0.01, p=.93, \eta_{\mathrm{p}}{ }^{2}=.05\right]$, suggesting that having the hands occupied by lightweight objects did not alter the sensitivity of the tactile duration judgments.

\section{Experiment 4}

The findings of Experiment 3 are consistent with the view that the tactile-duration expansion in the approaching-movement condition is attributable to the action context induced by real visual movement events, rather than to an attentional modulation. However, the real movements could also be associated with an increase in arousal. That is, an increase in arousalrather than the movement affordances themselves - might be the cause of the time expansion. To distinguish between these alternative accounts, in Experiment 4 we compared the arousal levels, measured in terms of SCRs, among the approachingmovement hands-occupied, approaching-movement handsfree, and static baseline (half hands-free and half hands-occupied) conditions (i.e., exactly the same conditions as were implemented in Exp. 3).

Figure 5 presents the psychometric curves for the various experimental conditions, along with the respective mean PSEs of the temporal bisection performance. The mean PSEs $( \pm 95 \% \mathrm{CI})$ were $397 \pm 23,410 \pm 26$, and $418 \pm 22 \mathrm{~ms}$ for the approaching-movement hands-free, approachingmovement hands-occupied, and static-baseline conditions, respectively. A one-way repeated measures ANOVA revealed the effect of movement type to be significant, $F(2,18)=5.84$, $p<.05, \eta_{\mathrm{p}}{ }^{2}=.39$. Follow-up Holm-Bonferroni tests indicated that the subjective tactile duration was significantly longer in the approaching-movement hands-free condition than in the static baseline conditions (mean difference: $21 \mathrm{~ms}$, corrected $p$ $=.03$ ), and was marginal significantly longer than in the approaching-movement hands-occupied condition (mean differences: $13 \mathrm{~ms}$, corrected $p=.06$ ), but that there was no difference between the approaching-movement hands-occupied and baseline conditions (mean difference: $8 \mathrm{~ms}$, corrected $p=.36$ ). Scaled JZS B.F.s indicated anecdotal difference between the approaching-movement hands-free and approaching-movement hands-occupied conditions (JZS B.F. $=0.37$ ) and strong difference between the approachingmovement hands-free and baseline conditions (JZS B.F. = 0.09 ), but were in favor of the null hypothesis for the approaching-movement hands-occupied versus baseline conditions (JZS B.F. = 2.18). Note that the mean differences in PSEs among the three conditions were relatively smaller than in the previous experiments, which was likely owing to the two SCR sensors attached to the index and middle fingers, which might have further inhibited automatic reaction tendencies in the movement conditions.

As in Experiment 3, a one-way repeated measures ANOVA of the JNDs revealed no effect of movement type on the discrimination sensitivity of the duration judgments, $F(2,18)=$ $0.28, p=.76, \eta_{\mathrm{p}}{ }^{2}=.03$.

The subjective SAM ratings showed that the two approaching-movement conditions (i.e., hands-occupied and hands-free) were rated as being slightly higher in arousal (5.6 and 5.5 for the hands-occupied and hands-free conditions, respectively) than the static baseline $(3.7), F(2,18)=16.51$, $p<.01, \eta_{\mathrm{p}}{ }^{2}=.65$; however, no difference was apparent between the two approaching-movement conditions $(p=.59)$. The differences in (rated) subjective arousal were not corroborated by the physiological data: A one-way repeated measures ANOVA failed to reveal any significant modulation of the SCRs, $F(2,18)=1.15, p=.34, \eta_{\mathrm{p}}{ }^{2}=.11$. This pattern suggests that, whereas the approaching ball movement had some impact on the subjective level of arousal, this was not associated with a physiological change in arousal. Importantly, the ratings did not differ between the two (hands-occupied and hands-free) approaching-movement conditions, indicating that the differential effects on the duration judgments between the two movement conditions cannot be attributed to differences in subjective arousal.

\section{General discussion}

The aim of the present study was to examine whether a taskirrelevant visual action context could cause a time distortion in tactile duration judgments. Participants were asked to judge the duration of a vibrotactile stimulus while watching an irrelevant swinging ball. We hypothesized that real ball movements might automatically elicit an appropriate action 
schema, even when participants were instructed not to make any explicit response (such as grasping) to the ball. The results indicated that monitoring task-irrelevant real ball movements with strong $\mathrm{S}-\mathrm{R}$ associations expanded tactile duration judgments relative to a static baseline condition, provided that the hands were not occupied. Participants markedly overestimated the tactile duration when the ball was approaching relative to when it was moving laterally or was static (Exp. 1). When the hand was placed at the middle path of the approaching and receding movements, providing comparable S-R associations, both conditions led to a comparable expansion of subjective tactile duration (Exp. 2). Most interestingly, the duration expansion engendered by observing an approaching ball was greatly reduced when participants held two lightweight objects in their hands (Exps. 3 and 4).

Previous studies had shown that a movement event is perceived as lasting longer than a static event of the same physical duration (Brown, 1995; Kanai, Paffen, Hogendoorn, \& Verstraten, 2006; Kaneko \& Murakami, 2009). It has been argued that a speeding up of the visual "internal clock" by moving objects provides an ecological advantage for the prevention of collisions, avoidance of predators, and so forth (Kaneko \& Murakami, 2009). It should be noted that in those studies, duration expansion was directly related to the moving stimuli, and judgments were made within the same, visual modality. In our study, by contrast, the main task was to estimate duration in the tactile modality, and the ball movement was a task-irrelevant visual event that bore no causal relation to the tactile stimulation. Given this, our findings go beyond previous reports by indicating that not only the perceived duration of the movement event itself, but also those of other, action-irrelevant events such as tactile stimulation may be affected.

It might be argued that these effects were due to the ball movement inducing a general arousal effect, since previous studies had shown that high-contrast or high-arousal stimuli speed up the internal pacemaker, expanding the subjective durations of those stimuli (Droit-Volet, Brunot, \& Niedenthal, 2004; Penton-Voak, Edwards, Percival, \& Wearden, 1996). However, this is unlikely to account for our results, because we found no physiological indications of increased arousal in the movement conditions (Exp. 4); that is, the ball swing was not experienced as a highly arousing stimulus, capable of speeding up the internal pacemaker. Even though the SAM subjective rating values were slightly higher in the two approaching-movement conditions than in the static condition, this increased subjective arousal cannot explain the differential subjective durations between the two approaching-movement (i.e., hands-occupied vs. hands-free) conditions (Exp. 3) and the lack of a difference between the approaching-movement hands-occupied and baseline conditions (Exp. 4). In this regard, our findings are consistent with the conclusions from saccadic chronostasis studies - namely, that the arousal induced by an action or movement is not a critical factor per se for the expansion of subjective duration (Yarrow et al., 2001; Yarrow et al., 2004).

One key factor that can influence subjective time perception is attentional modulation (Block \& Zakay, 1997; Zakay \& Block, 1997). A classic phenomenon-namely, that "time flies when you are having fun, and time drags when you are having trouble" (e.g., Droit-Volet \& Meck, 2007; Gable \& Poole, 2012) - is partly due to attentional and arousal modulations. According to attentional-gate theory (Block \& Zakay, 1997; Zakay \& Block, 1997), attentional resources are divided between timing and nontiming processes. When attention is engaged by some nontiming event, such as the context of a fun story you are reading, the timing process for estimating the reading time is temporally halted, leading to a subjective time contraction. An irrelevant stimulus, such as the ball movement in our study, might give rise to an analogous diversion (or disengagement) of attention from the tactile temporal process. If this were the case, we should have observed a subjective duration contraction for tactile temporal judgments. However, in the present experiments (hands-free conditions), we did not find duration contraction but observed the opposite: a subjective duration expansion. In terms of the attentional-gate theory, this would mean that the visual movement did not draw attention away from the tactile modality, at least not for tactile temporal processing. Additional evidence against attentional capture by the visual event is provided by the fact that the sensitivity of temporal bisection was unreduced in the movement as compared to the static conditions in any of four experiments. $^{2}$

Thus, instead, we propose that the real ball swing may spontaneously induce action preparation (e.g., preparation for manual grasping or collision avoidance), even though no explicit action is (to be) made (Fotowat \& Gabbiani, 2011), in that motor activation is likely to alter subjective time estimates. Consistent with our suggestion of an implicit reaction induced by the automatic activation of an S-R association, previous studies have confirmed that viewing objects with action meanings can prime manual responses related to those objects (Tucker \& Ellis, 2004); such priming effects have been observed in premotor and motor cortex, parietal visuomotor cortex, and the supplementary motor area (Bensmaïa, Killebrew, \& Craig, 2006; Grezes \& Decety, 2002; Kiefer, Sim, Helbig, \& Graf, 2011; Sim, Helbig, Graf, \& Kiefer, 2014). The intrinsic perception-action links revealed by these studies constitute a core notion of embodied cognition, which posits that perception and cognition can be appropriately understood only in terms of sensorimotor interaction (Engel et al., 2013; Varela, Thompson, \& Rosch, 1991). The

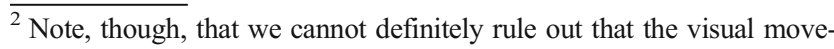
ment did capture attention, without however impeding tactile temporal processing, due to the simplicity of the required duration judgment.
} 
embodiment view has recently also been introduced in time perception (for reviews, see Droit-Volet et al., 2013; Effron et al., 2006; Gable \& Poole, 2012), providing a theoretical framework for our proposal of a functional linkage between action context and time perception. According to embodied timing (Craig, 2009; Droit-Volet et al., 2013; Effron et al., 2006; Niedenthal, 2007), subjective duration is based on both the sensory encoding of external events and the internal sensorimotor and bodily states during the judgment process. Indeed, a recent functional magnetic resonance imaging (fMRI) study has shown that insular cortex, a region typically involved in the awareness of emotional and bodily states (Craig, 2009), is activated during the duration-encoding phase of a temporal reproduction task (Wittmann, Simmons, Aron, $\&$ Paulus, 2010). The findings of the present study favor and extend the embodiment view of time perception: They show that temporal processing in one modality (here, touch) can be influenced by task-irrelevant motor activation through the operation of $\mathrm{S}-\mathrm{R}$ associations (even though no explicit action is being made), with the influence depending on the urgency and possibility of interaction. For instance, the urgency of a reaction was, arguably, higher for the approaching ball swing than for the lateral swing, and was similar for the approaching and receding swings in the present study. As a consequence, the tactile duration was perceived as being longer in the approaching- than in the lateral-movement condition, but the same as in the receding-movement condition (both approaching and receding movements provided equal opportunities for interaction). In addition, the weak S-R association with the lateral ball may be a cause for its marginal effect relative to the static conditions. Furthermore, when participants held two lightweight objects in their hands under conditions of approaching movement, the possibility of manually reacting to the ball was limited, and the motor activation (using the hands) might have been inhibited. As a result, the tactile expansion was greatly reduced in the approachingmovement hands-occupied condition (Exps. 3 and 4). Note that in Experiment 4, participants' fingers were tied to SCR sensors, which might have acted as an inhibitor that reduced the subjective duration expansion (relative to simply holding objects in Exp. 3) in the approaching-movement hands-free condition. Importantly, however, we found no difference in tactile duration judgments between the static hands-free and hands-occupied conditions (Exp. 3). These findings have several implications: First, both the differential effects on subjective duration between the approaching-movement conditions (hands-free vs. hands-occupied) and between the approaching- and lateral-movement conditions may be indicative of the roles of action preparation and its possibility, rather than merely reflecting the (abstract) representation of objects with action meanings (Barsalou, 2008; Kiefer \& Barsalou, 2013; Kiefer \& Pulvermüller, 2012). Second, the reduced effect in the approaching-movement hands-occupied condition also suggests that visual looming and/or a distance effect seems not to be the main factor for tactile-duration expansion, given that the movement settings (visual size and travelling distance) were exactly the same in the two approaching-movement (hands-free and hands-occupied) conditions. Third, the null difference in tactile-duration estimates between the static hands-free and hands-occupied conditions indicates that the subjective duration expansion observed in the ball movement conditions requires strong action context, rather than merely the presence of an affordance (Helbig, Graf, \& Kiefer, 2006).

One might ask why the implicit motor activation induced by the real ball swing (i.e., approaching and receding movements) could expand, rather than contract, the subjective tactile duration. Overestimation of target durations caused by action or action context has been demonstrated in various paradigms, such as during saccadic or manual movements (Yarrow et al., 2001; Yarrow \& Rothwell, 2003), simple duration reproduction (Ganzenmüller, Shi, \& Müller, 2012; Shi, Ganzenmüller, \& Müller, 2013), or manual preparation (Hagura et al., 2012). The idea that the various paradigms tap into different underlying mechanisms might explain why the accounts proposed for duration expansion are divergent. Duration expansion of an event that immediately follows an action has been interpreted in terms of a compensatory mechanism, in which the onset of the event is captured by the onset of the action (Yarrow et al., 2001); by contrast, expansion of the reproduced duration of an auditory event has been attributed to modality-specific "internal clocks," with the auditory clock ticking faster than other modality-specific clocks (Ganzenmüller et al., 2012; Shi, Ganzenmüller, \& Müller, 2013). It has also been suggested that activity induced by movement preparation in the motor cortices is linked to the initiation of dynamic movement activity (Churchland, Cunningham, Kaufman, Ryu, \& Shenoy, 2010), as well as to the facilitation of perceptual processing (Hagura et al., 2012). Similarly, implicit motor activation induced by nearby moving stimuli with strong interactions with the body may increase the speed of somatosensory processing, such that the real action, if it becomes necessary, can be targeted more precisely-thus yielding a greater benefit for survival. The duration expansion of tactile events observed in the present study may reflect such facilitated sensory processing induced by motor activation. This view favors the notion of embodied time perception (Craig, 2009), which proposes that altering bodily states (e.g., here, motor activation) changes the temporal resolution of the "moment" representation of the 
sentient self, a building block of time perception. It is worth noting that action does not always expand perceived duration. For example, when an action has a causal effect, the gap between the action and its consequent effects is shortened, which is attributed to "intentional (action-effect) binding" (Haggard \& Clark, 2003; Haggard, Clark, \& Kalogeras, 2002). However, this type of shortening effect is specific to causal events and is induced by the anticipatory awareness of action effects (Haggard \& Clark, 2003; Moore \& Haggard, 2008) or by general causal binding (Buehner, 2012; Buehner \& Humphreys, 2009).

Finally, note that the present study is potentially limited, in that activation of S-R associations was only manipulated using ball movements within reachable space (though Ball \& Tronick, 1971, showed an automatic avoiding reaction to a real approaching object in infants), and the strength of the associations, rather than being assessed directly, was only inferred from their (differential) effects on duration judgments. However, when compared to alternative accounts framed in terms of visual-looming, attention, and/or arousal effects, the embodiment framework arguably provides the better predictive power for our overall pattern of our results. Nevertheless, in order to corroborate our conclusions, future, complementary work should attempt to examine au- tomatic $\mathrm{S}-\mathrm{R}$ effects using physiological measures (e.g., electrocardio- or electroencephalography), so as to establish a more direct link between implicit reactions and time perception, and to examine different degrees of "interaction"- for instance, by comparing "near-body" with "far-body" movements. Further research should also examine whether crossmodal duration expansion by action context is specific to the tactile modality, or whether it can be generalized to other modalities, such as audition.

In conclusion, our results provide new evidence that task-irrelevant action contexts induced by seeing a real moving (approaching or receding) object expand tactile duration judgments. When induction of this context is physically inhibited, subjective expansion of tactile durations is greatly reduced. These findings favor an embodiment view of time perception (Craig, 2009; Droit-Volet et al., 2013; Effron et al., 2006; Niedenthal, 2007), according to which action preparation is likely to increase the speed of sensory processing (Hagura et al., 2012) and, consequently, to expand subjective time in the sensorimotor loop. Our findings suggest that subjective duration expansion by action contexts is not limited to the action itself or to events relating to action goals, but is also found for task-irrelevant motor activation occurring in the sensorimotor loop.

\section{Appendix}

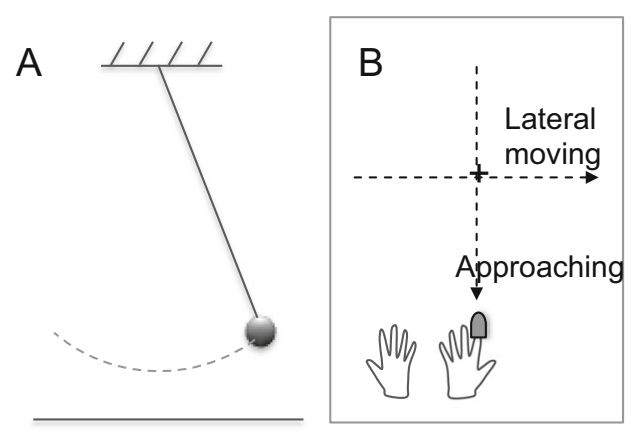

Fig. 1 Schematic illustrations of the setup used in the four experiments. (a) A pendulum swing was constructed with a metal ball. (b) In Experiment 1, participants placed their two hands, one next to the other, such that the right hand was close to the near endpoint of the maximum pendulum swing in the approaching-movement condition; the distance from the right index fingertip to the center of a fixation marker (which was located just under the equilibrium position of the ball pendulum) was $55 \mathrm{~cm}$. (c) In Experiment 2, participants placed their hands such that the right hand just rested on the fixation marker (the left hand was comfortably at rest next to the

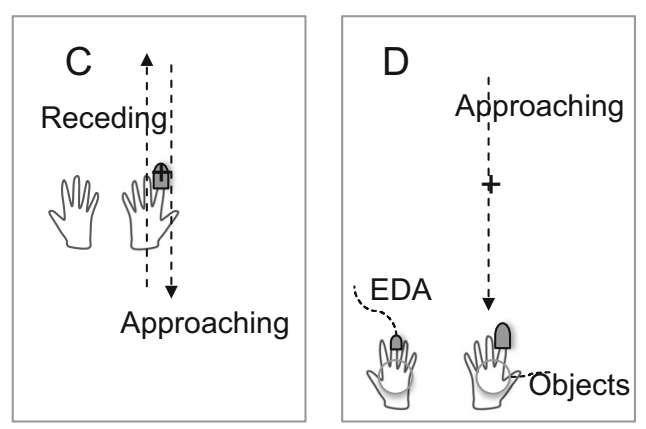

right hand); the distance from the participants' face/body to the fixation center was $45 \mathrm{~cm}$. (d) In Experiments 3 and 4, participants placed their hands again such that the right hand was close to the near endpoint of the maximum pendulum swing in the approaching-movement condition. In the hands-occupied blocks, two light objects were placed on the participants' palms throughout the duration of the trial blocks. In Experiment 4, two skin conductance response sensors were attached to the left-hand index and middle fingers to measure electrodermal activity (EDA). The distance from the right index fingertip to the fixation center was $39 \mathrm{~cm}$ 


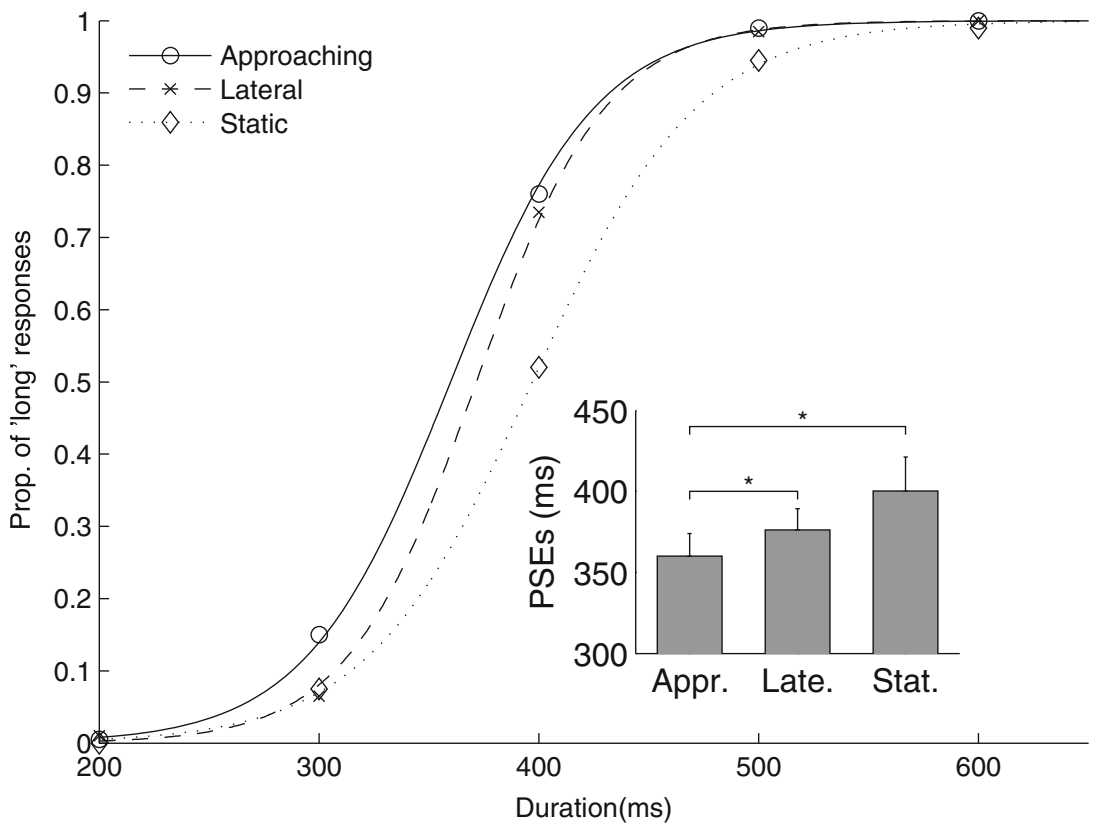

Fig. 2 Results of Experiment 1. Mean proportions of "long" responses in the tactile-duration bisection task, and the fitted psychometric functions, are plotted against the probe durations for the three visual-movement

conditions. The inset figure shows the mean points of subjective equality (PSEs) and the related $95 \%$ confident intervals (CIs) for the three conditions. ${ }^{*} p<.05$

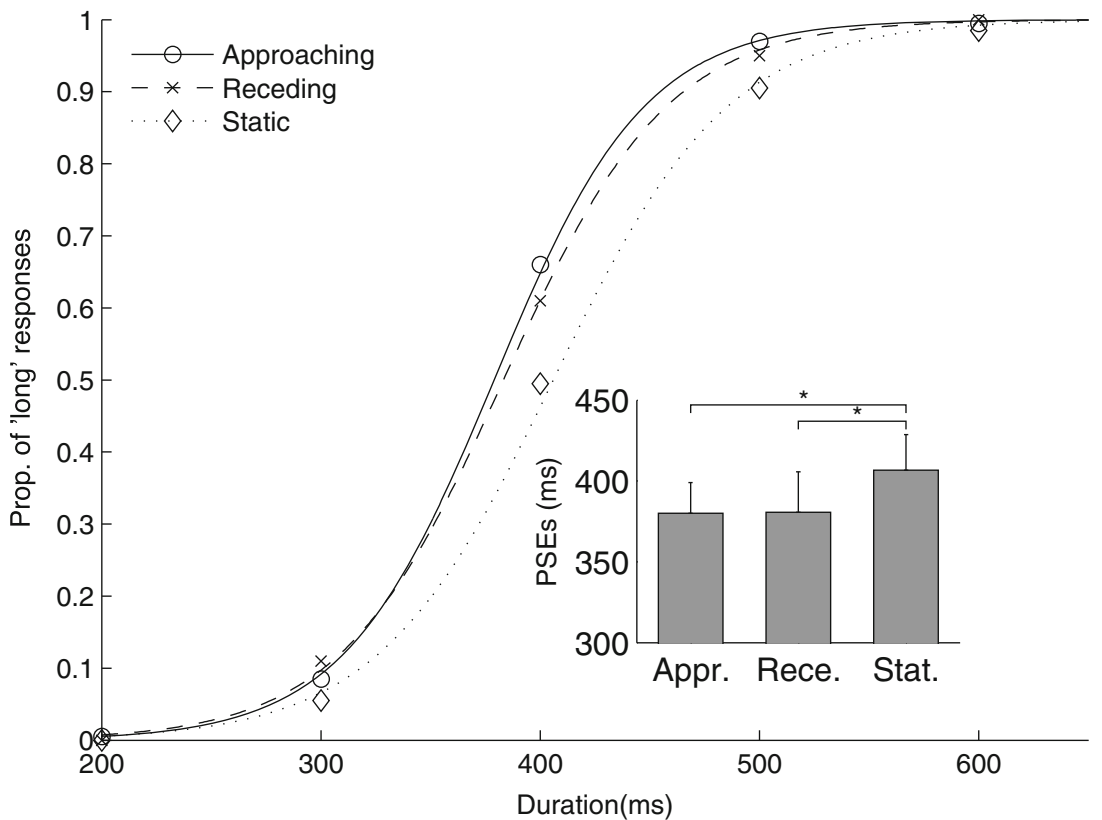

Fig. 3 Results of Experiment 2. Mean proportions of "long" responses in the tactile-duration bisection task, and the fitted psychometric functions, are plotted against the probe durations for the three visual-movement

conditions. The inset figure shows the mean PSEs and the related $95 \%$ CIs for the three conditions, with the approaching- and receding- movement conditions differing significantly from the static baseline. ${ }^{*} p<.05$ 


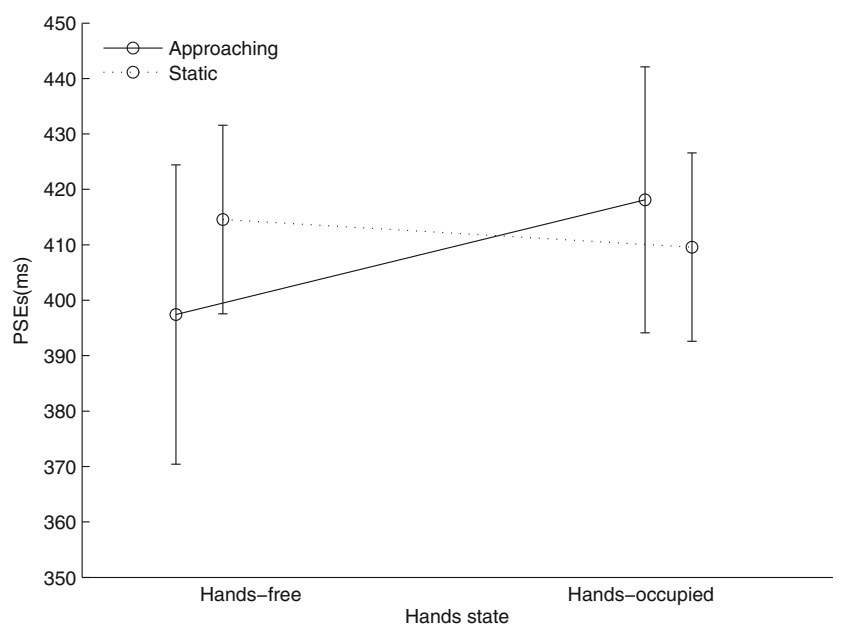

Fig. 4 Results of Experiment 3. The PSEs and the related $95 \%$ CIs are plotted against hand state (hands-free vs. hands-occupied) for the approaching-movement (solid line) and static (dotted line) conditions. The two lines cross each other, showing a significant interaction effect, $p<.05$

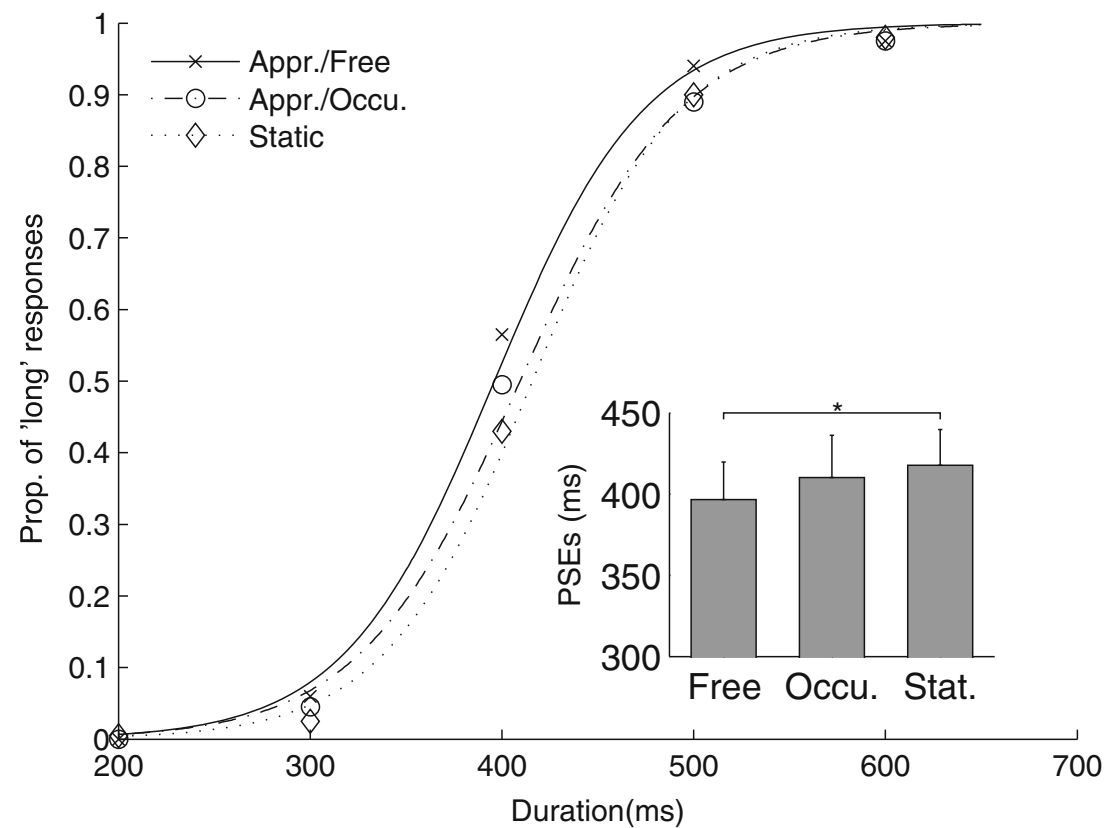

Fig. 5 Results of Experiment 4. The mean proportions of "long" responses in the tactile-duration bisection task, and the fitted psychometric functions, are plotted against the probe durations for the three ball- movement conditions: approaching hands-free, approaching hands-occupied, and static. The inset figure shows the mean PSEs and the related $95 \%$ CIs for the three conditions. ${ }^{*} p<.05$ 


\section{References}

Ball, W., \& Tronick, E. (1971). Infant responses to impending collision: Optical and real. Science, 171, 818-820. doi:10.1126/science.171. 3973.818

Barsalou, L. W. (2008). Grounded cognition. Annual Review of Psychology, 59, 617-645. doi:10.1146/annurev.psych.59.103006. 093639

Bensmaïa, S. J., Killebrew, J. H., \& Craig, J. C. (2006). Influence of visual motion on tactile motion perception. Journal of Neurophysiology, 96, 1625-1637. doi:10.1152/jn.00192.2006

Block, R. A., \& Zakay, D. (1997). Prospective and retrospective duration judgments: A meta-analytic review. Psychonomic Bulletin \& Review, 4, 184-197. doi:10.3758/BF03209393

Bradley, M. M., \& Lang, P. J. (1994). Measuring emotion: The selfassessment manikin and the semantic differential. Journal of Behavior Therapy and Experimental Psychiatry, 25, 49-59. doi: 10.1016/0005-7916(94)90063-9

Brown, S. (1995). Time, change, and motion: The effects of stimulus movement on temporal perception. Perception \& Psychophysics, 57, 105-116. doi:10.3758/BF03211853

Buehner, M. J. (2012). Understanding the past, predicting the future: Causation, not intentional action, is the root of temporal binding. Psychological Science, 23, 1490-1497. doi:10.1177/ 0956797612444612

Buehner, M. J., \& Humphreys, G. R. (2009). Causal binding of actions to their effects. Psychological Science, 20, 1221-1228. doi:10.1111/j. 1467-9280.2009.02435.x

Churchland, M. M., Cunningham, J. P., Kaufman, M. T., Ryu, S. I., \& Shenoy, K. V. (2010). Cortical preparatory activity: Representation of movement or first cog in a dynamical machine? Neuron, 68,387 400. doi:10.1016/j.neuron.2010.09.015

Clark, A. (1999). An embodied cognitive science? Trends in Cognitive Sciences, 3, 345-351. doi:10.1016/S1364-6613(99)01361-3

Craig, A. D. (2009). Emotional moments across time: A possible neural basis for time perception in the anterior insula. Philosophical Transactions of the Royal Society B, 364, 1933-1942. doi:10. 1098/rstb.2009.0008

Droit-Volet, S., Brunot, S., \& Niedenthal, P. M. (2004). Perception of the duration of emotional events. Cognition and Emotion, 18, 849-858. doi:10.1080/02699930341000194

Droit-Volet, S., Fayolle, S., Lamotte, M., \& Gil, S. (2013). Time, emotion and the embodiment of timing. Timing and Time Perception, 1, 99126

Droit-Volet, S., \& Gil, S. (2009). The time-emotion paradox. Philosophical Transactions of the Royal Society B, 364, 19431953. doi:10.1098/rstb.2009.0013

Droit-Volet, S., \& Meck, W. H. (2007). How emotions colour our perception of time. Trends in Cognitive Sciences, 11, 504-513. doi:10. 1016/j.tics.2007.09.008

Effron, D. A., Niedenthal, P. M., Gil, S., \& Droit-Volet, S. (2006). Embodied temporal perception of emotion. Emotion, 6, 1-9. doi: 10.1037/1528-3542.6.1.1

Engel, A. K., Maye, A., Kurthen, M., \& Konig, P. (2013). Where's the action? The pragmatic turn in cognitive science. Trends in Cognitive Sciences, 17, 202-209. doi:10.1016/j.tics.2013.03.006

Fotowat, H., \& Gabbiani, F. (2011). Collision detection as a model for sensory-motor integration. Annual Review of Neuroscience, 34, 119. doi:10.1146/annurev-neuro-061010-113632

Gable, P. A., \& Poole, B. D. (2012). Time flies when you're having approach-motivated fun: Effects of motivational intensity on time perception. Psychological Science, 23, 879-886. doi:10.1177/ 0956797611435817

Ganzenmüller, S., Shi, Z., \& Müller, H. J. (2012). Duration reproduction with sensory feedback delay: Differential involvement of perception and action time. Frontiers in Integrative Neuroscience, 6, 95. doi:10. 3389/fnint.2012.00095

Gibbon, J., \& Church, R. M. (1990). Representation of time. Cognition, 37, 23-54. doi:10.1016/0010-0277(90)90017-E

Gibbon, J., Church, R. M., \& Meck, W. H. (1984). Scalar timing in memory. Annals of the New York Academy of Sciences, 423, 52 77. doi:10.1111/j.1749-6632.1984.tb23417.x

Grezes, J., \& Decety, J. (2002). Does visual perception of object afford action? Evidence from a neuroimaging study. Neuropsychologia, 40, 212-222. doi:10.1016/S0028-3932(01)00089-6

Grondin, S., Laflamme, V., \& Gontier, É. (2014). Effect on perceived duration and sensitivity to time when observing disgusted faces and disgusting mutilation pictures. Attention, Perception, \& Psychophysics, 76, 1522-1534. doi:10.3758/s13414-014-0682-7

Haggard, P., \& Clark, S. (2003). Intentional action: Conscious experience and neural prediction. Consciousness and Cognition, 12, 695-707. doi:10.1016/S1053-8100(03)00052-7

Haggard, P., Clark, S., \& Kalogeras, J. (2002). Voluntary action and conscious awareness. Nature Neuroscience, 5, 382-385. doi:10. $1038 / \mathrm{nn} 827$

Hagura, N., Kanai, R., Orgs, G., \& Haggard, P. (2012). Ready steady slow: Action preparation slows the subjective passage of time. Proceedings of the Royal Society B, 279, 4399-4406. doi:10.1098/ rspb.2012.1339

Helbig, H. B., Graf, M., \& Kiefer, M. (2006). The role of action representations in visual object recognition. Experimental Brain Research, 174, 221-228. doi:10.1007/s00221-006-0443-5

Hodinott-Hill, I., Thilo, K. V., Cowey, A., \& Walsh, V. (2002). Auditory chronostasis: hanging on the telephone. Current Biology, 12, 17791781. doi:10.1016/S0960-9822(02)01219-8

Kanai, R., Paffen, C. L., Hogendoorn, H., \& Verstraten, F. A. (2006). Time dilation in dynamic visual display. Journal of Vision, 6(12), 1421-1430. doi:10.1167/16.12.8

Kaneko, S., \& Murakami, I. (2009). Perceived duration of visual motion increases with speed. Journal of Vision, 9(7), 14. doi:10.1167/9.7.14

Kiefer, M., \& Barsalou, L. W. (2013). Grounding the human conceptual system in perception, action, and internal states. In W. Prinz, M. Beisert, \& A. Herwig (Eds.), Action science: Foundations of an emerging discipline (pp. 381-407). Cambridge, MA: MIT Press.

Kiefer, M., \& Pulvermüller, F. (2012). Conceptual representations in mind and brain: Theoretical developments, current evidence and future directions. Cortex, 48, 805-825. doi:10.1016/j.cortex.2011. 04.006

Kiefer, M., Sim, E. J., Helbig, H., \& Graf, M. (2011). Tracking the time course of action priming on object recognition: Evidence for fast and slow influences of action on perception. Journal of Cognitive Neuroscience, 23, 1864-1874. doi:10.1162/jocn.2010.21543

Matthews, W. J. (2011). Stimulus repetition and the perception of time: The effects of prior exposure on temporal discrimination, judgment, and production. PloS One, 6, 19815. doi:10.1371/journal.pone. 0019815

Moore, J., \& Haggard, P. (2008). Awareness of action: Inference and prediction. Consciousness and Cognition, 17, 136-144. doi:10. 1016/j.concog.2006.12.004

Morrone, M. C., Ross, J., \& Burr, D. (2005). Saccadic eye movements cause compression of time as well as space. Nature Neuroscience, 8 , 950-954. doi:10.1038/nn1488

Nather, F. C., Bueno, J. L. O., Bigand, E., \& Droit-Volet, S. (2011). Time changes with the embodiment of another's body posture. PloS One, 6, 19818. doi:10.1371/journal.pone.0019818

Niedenthal, P. M. (2007). Embodying emotion. Science, 316, 1002-1005. doi:10.1126/science. 1136930

Orgs, G., Bestmann, S., Schuur, F., \& Haggard, P. (2011). From body form to biological motion: The apparent velocity of human movement biases subjective time. Psychological Science, 22, 712-717. doi:10.1177/0956797611406446 
Pariyadath, V., \& Eagleman, D. M. (2008). Brief subjective durations contract with repetition. Journal of Vision, 8(11), 11-16. doi:10. $1167 / 8.16 .11$

Park, J., Schlag-Rey, M., \& Schlag, J. (2003). Voluntary action expands perceived duration of its sensory consequence. Experimental Brain Research, 149, 527-529. doi:10.1007/s00221-003-1376-x

Penton-Voak, I. S., Edwards, H., Percival, A., \& Wearden, J. H. (1996). Speeding up an internal clock in humans? Effects of click trains on subjective duration. Journal of Experimental Psychology: Animal Behavior Processes, 22, 307-320. doi:10.1037/0097-7403.22.3.307

Shi, Z., Church, R. M., \& Meck, W. H. (2013a). Bayesian optimization of time perception. Trends in Cognitive Sciences, 17, 556-564. doi:10. 1016/j.tics.2013.09.009

Shi, Z., Ganzenmüller, S., \& Müller, H. J. (2013b). Reducing bias in auditory duration reproduction by integrating the reproduced signal. PloS One, 8, 62065. doi:10.1371/journal.pone.0062065

Shi, Z., Jia, L., \& Müller, H. J. (2012). Modulation of tactile duration judgments by emotional pictures. Frontiers in Integrative Neuroscience, 6, 24. doi:10.3389/fnint.2012.00024

Sim, E. J., Helbig, H. B., Graf, M., \& Kiefer, M. (2014). When action observation facilitates visual perception: Activation in visuo-motor areas contributes to object recognition. Cerebral Cortex. doi:10. 1093/cercor/bhu087.

Tucker, M., \& Ellis, R. (2004). Action priming by briefly presented objects. Acta Psychologica, 116, 185-203. doi:10.1016/j.actpsy.2004.01.004

van Wassenhove, V., Wittmann, M., Craig, A. D., \& Paulus, M. P. (2011). Psychological and neural mechanisms of subjective time dilation. Frontiers in Neuroscience, 5, 56. doi:10.3389/fnins.2011.00056

Varela, F. J., Thompson, E., \& Rosch, E. (1991). The embodied mind: Cognitive science and human experience. Cambridge, MA: MIT Press.
Wittmann, M. (2013). The inner sense of time: How the brain creates a representation of duration. Nature Reviews Neuroscience, 14, $217-$ 223. doi: $10.1038 / \mathrm{nrn} 3452$

Wittmann, M., Simmons, A. N., Aron, J. L., \& Paulus, M. P. (2010a). Accumulation of neural activity in the posterior insula encodes the passage of time. Neuropsychologia, 48, 3110-3120. doi:10.1016/j. neuropsychologia.2010.06.023

Wittmann, M., \& van Wassenhove, V. (2009). The experience of time: Neural mechanisms and the interplay of emotion, cognition and embodiment. Philosophical Transactions of the Royal Society B, 364, 1809-1813. doi:10.1098/rstb.2009.0003

Wittmann, M., van Wassenhove, V., Craig, A. D., \& Paulus, M. P. (2010b). The neural substrates of subjective time dilation. Frontiers in Human Neuroscience, 4, 2. doi:10.3389/neuro.09.002. 2010

Yamamoto, K., \& Miura, K. (2012). Time dilation caused by static images with implied motion. Experimental Brain Research, 223, 311319. doi:10.1007/s00221-012-3259-5

Yarrow, K., Haggard, P., Heal, R., Brown, P., \& Rothwell, J. C. (2001). Illusory perceptions of space and time preserve cross-saccadic perceptual continuity. Nature, 414, 302-305. doi:10.1038/35104551

Yarrow, K., Haggard, P., \& Rothwell, J. C. (2004). Action, arousal, and subjective time. Consciousness and Cognition, 13, 373-390. doi:10. 1016/j.concog.2003.10.006

Yarrow, K., \& Rothwell, J. C. (2003). Manual chronostasis: Tactile perception precedes physical contact. Current Biology, 13, 1134-1139. doi:10.1016/S

Zakay, D., \& Block, R. A. (1997). Temporal cognition. Current Directions in Psychological Science, 6, 12-16. doi:10.1111/14678721.ep11512604 